\title{
Le nouveau régionalisme métropolitain aux États-Unis
}

\author{
Éric Champagne ${ }^{1}$ \\ INRS-Urbanisation, Culture et Société
}

L'objectif de cet article est de décrire l'évolution récente des idées urbanistiques et des pratiques métropolitaines aux États-Unis. Dans ce pays, la dynamique de l'organisation municipale et métropolitaine se caractérise par l'absence de réformes structurelles majeures. Cependant, on constate depuis plusieurs années une vive effervescence dans le développement des idées urbanistiques portées par un mouvement qu'il convient de qualifier de "nouveau régionalisme métropolitain». L'article soutient que ce mouvement n'a pas su imposer concrètement un nouvel ordre institutionnel métropolitain mais qu'il a surtout favorisé une mobilisation d'acteurs régionaux autour des enjeux du développement et de la croissance métropolitaine.

Depuis le début des années $1990^{2}$ plusieurs provinces canadiennes ont initié des projets de réforme municipale de grande envergure ayant donné lieu à une actualisation des modes de gestion et de planification au sein des principales régions métropolitaines. Vers la fin des années 1990 et le début des années 2000, on assiste notamment à des réformes structurelles majeures dans les deux plus grandes régions métropolitaines du Canada. D'abord le premier janvier 1998, le gouvernement provincial de l'Ontario concrétise la fusion des six composantes municipales du gouvernement métropolitain (Metro Toronto) en une seule municipalité. Le gouvernement de l'Ontario crée dans la même veine le Greater Toronto Services Board afin de faciliter la coordination municipale dans l'ensemble de la région métropolitaine. Le 20 décembre 2000 marque le début d'une réforme municipale majeure des principales régions métropolitaines québécoises avec l'adoption par le gouvernement du Québec du projet de loi 170. Ce projet de loi prévoit notamment la création de la nouvelle ville de Montréal en regroupant les 28 municipalités de la Communauté urbaine de Montréal. Afin de favoriser la coordination des grandes fonctions métropolitaines le gouvernement du Québec crée une instance régionale, la Communauté métropolitaine de Montréal (CMM), couvrant le territoire complet de la région métropolitaine de recensement.

Pendant cette même période aux États-Unis, malgré des tendances spatiales similaires à celles du Canada et une structure de relations intergouvernementales relativement comparable, la dynamique de l'organisation municipale et métropolitaine se caractérise au contraire par l'absence de réformes structurelles majeures. En dépit de cela, on constate une vive effervescence dans le développement des idées urbanistiques ainsi que dans l'élaboration d'un ensemble d'initiatives visant des réformes métropolitaines portées par un mouvement qu'il convient de qualifier de «nouveau régionalisme métropolitain $»^{3}$. Ce mouvement repose sur un ensemble de nouvelles représentations théoriques apparaissant au début des années 1990 en réponse aux tendances spatiales marquées par l'étalement urbain ainsi qu'en réaction aux approches existantes de l'organisation municipale et métropolitaine. Cette école de pensée s'articule autour de la notion d'interdépendance de la ville centre et des banlieues ainsi que du maintien de la compétitivité économique dans le contexte de la mondialisation tout en insistant sur l'importance de la viabilité sociale et environnementale du développement métropolitain. Cette approche vise, à terme, à mettre en œuvre des réformes métropolitaines afin d'adapter les structures de pouvoir au nouvel ordre économique et spatial. Si le mouvement du nouveau régionalisme n'aboutit pas comme au Canada à des réformes «structurelles » de l'organisation municipale et métropolitaine, nous montrons dans cet article qu'il conduit à de nouveaux modes de gouvernance influencés par la montée des acteurs non gouvernementaux dans la gestion des affaires publiques.

\section{L'organisation municipale aux États-Unis}

L'organisation municipale aux États-Unis relève directement des États et chacun peut, en principe, développer ses 
Tableau 1. Évolution des gouvernements locaux aux États-Unis, 1952-1997

\begin{tabular}{lrrrrrr}
\hline Type de gouvernement & $\mathbf{1 9 5 2}$ & $\mathbf{1 9 6 2}$ & $\mathbf{1 9 7 2}$ & $\mathbf{1 9 8 2}$ & $\mathbf{1 9 9 2}$ & $\mathbf{1 9 9 7}$ \\
\hline Comtés & 3052 & 3043 & 3044 & 3041 & 3043 & 3043 \\
Municipalités & 34009 & 35142 & 35508 & 35810 & 35935 & 36001 \\
Agences unifonctionnelles & 12340 & 18323 & 23885 & 28078 & 31555 & 34683 \\
\hline Total & 51353 & 58470 & 64409 & 68911 & 72525 & 75724 \\
\hline
\end{tabular}

Source: Bureau of the Census, Statistical Abstract of the United States, 1998.

propres traditions municipales. Chaque État possède une organisation municipale particulière avec des règles, des partages de responsabilités ou des systèmes fiscaux qui peuvent varier de l'un à l'autre. L'organisation municipale repose essentiellement sur trois types d'institutions : les municipalités, les comtés et les agences unifonctionnelles ${ }^{4}$ (tableau 1).

En 1997, on recensait 3043 comtés aux États-Unis et 36001 municipalités. Les comtés sont à l'origine des instances administratives des États ${ }^{5}$. Si leurs fonctions initiales visaient davantage à administrer régionalement les services publics de l'État, ils assument désormais un éventail de services de nature régionale comme le traitement des déchets, la santé publique, le transport public, la protection de l'environnement, etc. Le comté est ainsi devenu un véritable niveau supramunicipal aux États-Unis.

Une autre forme d'institution locale a pris une importance considérable au cours des dernières décennies aux États-Unis. Il s'agit des agences unifonctionnelles (special districts). Les agences unifonctionnelles sont généralement créées par une ou plusieurs municipalités afin d'assurer la fourniture de services locaux et régionaux; que ce soit dans la gestion ou la protection de l'environnement, le logement social, le développement communautaire, la protection des incendies, la gestion des égouts ou des aqueducs, les parcs et les loisirs, la santé et les hôpitaux, les bibliothèques, le transport en commun et les autres utilités publiques (ports, aéroports, etc.). Cette capacité d'intervention s'exprime entre autres par la possibilité de s'autofinancer par la tarification des services ou par l'imposition d'une taxe foncière autonome. De plus, comme les États limitent généralement le pouvoir d'endettement municipal, les agences unifonctionnelles sont parfois créées pour encadrer le financement des grands projets d'infrastructure ou de services régionaux. Le nombre d'agences unifonctionnelles est passé de 12340 à 34683 entre 1952 et 1997, de telle sorte que la dépendance envers elles pour la fourniture de services locaux et régionaux n'a cessé de grandir ${ }^{6}$.
Les municipalités, soucieuses de protéger leur autonomie locale, ont souvent vu dans le renforcement des comtés ou dans la création de nouvelles agences unifonctionnelles une protection contre les solutions plus lourdes de réformes métropolitaines comme par exemple le recours aux fusions municipales ou la création de nouvelles instances métropolitaines. Ainsi, l'évolution de l'organisation municipale aux États-Unis se caractérise par l'absence de réformes majeures des structures municipales.

\section{L'évolution de l'organisation municipale aux États-Unis se caractérise par l'absence de réformes majeures des structures municipales.}

Le caractère non interventionniste des États ne repose pas seulement sur la légalité constitutionnelle mais également sur les traditions politiques. Bien qu'ils en aient théoriquement le pouvoir, ces derniers évitent généralement de se mêler des affaires municipales. Tout spécialement s'il s'agit de réformes touchant les structures institutionnelles, les territoires des juridictions et les finances publiques. L'autonomie municipale est un principe politique devenu presque intouchable. Avec près de 76000 unités administratives locales à la grandeur du pays, les régions métropolitaines étasuniennes se caractérisent généralement par un environnement institutionnel complexe et des plus fragmenté.

\section{La mosaïque des approches métropolitaines et les conseils régionaux}

Plutôt que d'envisager des solutions structurelles comme les fusions municipales ou la création d'instances ou de gouvernements métropolitains, les réformes métropolitaines aux États-Unis ont surtout nécessité le recours à une mosaïque hétérogène d'approches afin de coordonner les services publics régionaux. Le renforcement des comtés et la création d'agences unifonctionnelles comptent parmi les mesures les plus communes permettant d'administrer 
certaines fonctions régionales tout en préservant l'autonomie et l'intégrité des municipalités. À cela s'ajoute toute une panoplie de mesures plus ou moins ad hoc qu'on peut associer avec la gouvernance du territoire métropolitain. Certaines régions ont adopté des procédures de partage de la taxe foncière (tax base sharing), la plupart se sont dotées d'organismes de promotion ou de développement économique et on retrouve également une série d'initiatives métropolitaines temporaires visant la mobilisation d'un ensemble d'acteurs autour d'un projet très spécifique.

L'univers institutionnel métropolitain aux États-Unis a surtout été marqué par l'établissement d'instances régionales qui ont souvent pris la forme de conseils régionaux? Ces conseils régionaux ont acquis une place relativement importante pour la coordination et la collaboration métropolitaine. À l'exception des régions de Minneapolis et de Portland dont les instances métropolitaines adoptent un caractère gouvernemental ou quasi gouvernemental ${ }^{8}$, les conseils régionaux ne font pas partie de l'appareil gouvernemental proprement dit. Il faut ajouter que ce sont principalement les programmes fédéraux de subventions dans les années 1960 et 1970 qui ont stimulé la multiplication des conseils régionaux dans presque toutes les régions métropolitaines. Typiquement, les conseils régionaux ont quatre fonctions :

1. ils élaborent des documents de planification stratégique ;

2. ils contribuent à l'émergence d'une vision régionale;

3. ils ont le mandat de coordonner la programmation de certaines dépenses d'immobilisations régionales du gouvernement fédéral ;

4. ils servent de lieu d'arbitrage pour les litiges entre municipalités.

Ces conseils régionaux ne sont généralement toutefois que des organismes de consultation et de concertation. Ils n'ont habituellement pas de pouvoir de taxation, ne disposent pas de pouvoirs formels pour mettre en œuvre les règlements ou les dispositions qu'ils adoptent et ne s'occupent généralement pas de fourniture directe de services publics. Les conseils régionaux constituent pour les municipalités une solution relativement flexible dans l'exercice des fonctions régionales tout en permettant de préserver leur autonomie politique. Il s'agit probablement de la formule la plus souple que l'on puisse envisager afin d'assurer une certaine forme de coordination métropolitaine. Les conseils régionaux n'ont le plus souvent qu'un statut consultatif et ont peu de pouvoir direct sur les décisions de municipalités.
Par conséquent, les conseils régionaux demeurent surtout une forme coopérative et volontaire de gouvernance métropolitaine plutôt qu'une forme institutionnelle permettant des interventions musclées. L'action des conseils régionaux demeure de ce fait limitée pour aborder les enjeux ou pour la résolution des problèmes métropolitains contemporains.

\section{Les conseils régionaux demeurent surtout une forme coopérative et volontaire de gouvernance métropolitaine plutôt qu'une forme institutionnelle permettant des interventions musclées.}

\section{Les porteurs du nouveau régionalisme}

Le nouveau régionalisme qui émerge au début des années 1990 découle d'une réponse face aux problèmes engendrés par une croissance urbaine non contrôlée et d'une insatisfaction à l'égard des modèles existants d'organisation municipale et métropolitaine. Ces modèles auraient échoué de quatre manières:

- à susciter une cohésion régionale et à diminuer la concurrence intra-métropolitaine ;

- à potentialiser la compétitivité des régions métropolitaines ;

- à résoudre les problèmes reliés à la croissance urbaine (étalement urbain, transport, environnement, ségrégation sociale entre les villes centres et les banlieues, etc.);

- à diminuer les disparités sociales entre les composantes métropolitaines.

Le nouveau régionalisme constitue d'abord une école de pensée dont les idées ont été reprises par un certain nombre d'acteurs sociaux, économiques ou politiques en vue de les transposer en actions concrètes. On observe d'ailleurs que ce mouvement est porté par trois types d'acteurs : les universitaires (intellectuels), les experts (conseillers) et les organisations nationales et régionales (tableau 2). Les penseurs académiques sont derrière le mouvement intellectuel qui a alimenté depuis environ une dizaine d'années un débat de fond sur le sort des agglomérations urbaines et surtout sur les conséquences négatives à long terme du style de développement métropolitain. Ces chercheurs ont élaboré un nouveau paradigme concernant le développement métropolitain en proposant une redéfinition du rôle des régions métropolitaines dans l'environnement national et mondial. Plusieurs de ces études 
ont apporté une contribution significative aux principaux arguments du nouveau régionalisme.

Parmi les experts, plusieurs sont à la fois des théoriciens et des militants du nouveau régionalisme sur la scène nationale. Ces individus ont généralement soit une position médiatique influente, soit une expérience pratique de l'univers des politiques publiques. Ils sont tantôt éditorialistes pour des journaux nationaux (Neil Peirce, Washington Post), ils ont tantôt une large expérience de politicien (Myron Orfield, législateur au Minnesota ou David Rusk, ancien maire de Albuquerque, NM). Ils font parfois partie de la haute fonction publique (Henry G. Cisneros, ancien Secrétaire du US. Housing and Urban Development) ou ils occupent un poste de haute direction au sein d'une des associations nationales qui foisonnent aux États-Unis (William R. Dodge ancien directeur du National Association of Regional Councils) ou d'un des nombreux groupes de réflexions (think-tank) étasunien (Bruce Katz est directeur du Brookings Institution Center on Urban and Metropolitan Policy). Les experts font la promotion d'initiatives métropolitaines diverses afin de freiner l'étalement urbain et limiter la croissance urbaine, de protéger l'environnement et les espaces vierges, de réduire la ségrégation sociale, de revitaliser les villes centres et les banlieues de première couronne et de mieux redistribuer la richesse en établissant un meilleur équilibre fiscal entre les municipalités les plus pauvres et les plus riches. La ligne n'est pas facile à tracer entre les experts et les universitaires, car les deux groupes contribuent à leur manière à définir un nouveau cadre d'analyse concernant le développement métropolitain. Certains peuvent facilement se retrouver dans les deux camps comme Larry Ledebur qui combine la carrière académique et celle de recherche politique pour le gouvernement fédéral et certaines associations nationales comme le National League of Cities, ou le Urban Institute's Economic Development Program.

Il existe enfin un ensemble d'organisations diverses qui œuvrent activement à l'échelle nationale ou à l'intérieur des régions métropolitaines afin de favoriser la mise en œuvre de réformes métropolitaines. Sur la scène nationale, on retrouve les associations représentant les conseils régionaux, les comtés, les municipalités ou les maires des États-Unis. Ces regroupements puisent leur légitimité de la représentativité que leur confère l'adhésion d'un grand nombre de membres répartis à la grandeur du pays, ce qui leur permet de prendre part aux débats sur les enjeux métropolitains. On retrouve également certains départements du gouvernement fédéral qui sont directement touchés par la problématique métropolitaine comme le Housing and Urban Development dont le mandat regarde le logement et le développement urbain. À cela s'ajoutent les influents groupes de réflexion étasuniens comme le Brooking Institute, le Lincoln Land Institute et le Urban Institute et certains réseaux d'intérêts qui se sont formés à la grandeur du pays afin de promouvoir le métropolitanisme.

\section{Tableau 2. Les acteurs du nouveau régionalisme}

\begin{tabular}{llll}
\hline Universitaires / intellectuels & Experts / conseillers & Organisations nationales et régionales \\
\hline $\begin{array}{l}\text { Les universitaires proposent } \\
\text { un nouveau paradigme concernant } \\
\text { le développement métropolitain }\end{array}$ & $\begin{array}{l}\text { Les experts contribuent } \\
\text { au mouvement en fournissant } \\
\text { des solutions métropolitaines }\end{array}$ & $\begin{array}{l}\text { Les organisations civiques, politiques ou associatives } \\
\text { militent concrètement en faveur de réformes métropolitaines } \\
\text { à l'échelle nationale ou régionale }\end{array}$ \\
Allan D. Wallis & Henry G. Cisneros & Organisations nationales & Organisations régionales \\
Anthony Downs & David Rusk & National Association & Associations d'affaires \\
Patricia Atkins & Myron Orfield & National Association & Groupes sociaux \\
Kathryn A. Foster & Neal Peirce & of Counties & et communautaires \\
Hank V. Savitch & William R. Dodge & National League of Cities / & Groupes écologistes \\
Ronald K. Vogel & Larry C. Ledebur & U.S. Conference of Mayors & Organismes de planification, \\
Larry C. Ledebur & William R. Barnes & US. Department of Housing & de développement \\
Joseph Persky & Bruce Katz & Brookings Institution / Unt Development & Agences de transport \\
Kenichi Ohmae & & Lincoln Land Institute / & Certaines municipalités / \\
Todd Swanstrom & & Urban Institute & Certains États \\
Richard Voith & & Citistates Group / & Médias
\end{tabular}


Sur la scène régionale, de nombreux groupes sont actifs sur cette question ; à commencer par les associations d'affaires qui ont tendance à voir les régions métropolitaines comme un ensemble économique indivisible. Les groupes sociaux et communautaires insistent plutôt sur les inégalités engendrées par le développement métropolitain incontrôlé. Les groupes environnementaux critiquent pour leur part les conséquences écologiques de la croissance urbaine et les orientations dans le domaine du transport. Évidemment, les organismes de planification, d'aménagement et de développement régionaux incluant les conseils régionaux ont généralement eu tendance à appuyer les initiatives métropolitaines. Certaines municipalités centrales et de banlieue ont commencé à s'intéresser aux enjeux métropolitains. Notamment les banlieues de première couronne qui partagent généralement beaucoup de points communs avec les villes centres sur les enjeux du développement économique et territorial. Bien qu'ils aient été réticents à initier des réformes municipales structurelles, les États ne sont pas totalement indifférents au discours du nouveau régionalisme. L'efficacité des dépenses publiques et les enjeux de la compétitivité économique sont les éléments du nouveau régionalisme les plus souvent retenus par les États dans l'exécution de nouvelles politiques. Enfin, les médias (notamment les journaux) ont souvent adopté un parti pris favorable au discours du nouveau régionalisme dans plusieurs régions étasuniennes. En somme, le nouveau régionalisme est porté par un ensemble d'organisations aux intérêts variés mais convergents qui font la promotion d'un nouvel ordre du jour métropolitain.

\section{Le nouveau régionalisme est porté par un ensemble d'organisations aux intérêts variés mais convergents qui font la promotion d'un nouvel ordre du jour métropolitain.}

\section{Les arguments du nouveau régionalisme métropolitain}

Les solutions proposées par le nouveau régionalisme ne sont pas toujours nouvelles. C'est plutôt dans le discours et les arguments invoqués que le nouveau régionalisme a quelque chose d'inédit. Sur le plan de l'argumentation, il est davantage préoccupé par la qualité du développement métropolitain et la compétitivité économique régionale contrairement aux régionalismes précédents dont l'objectif visait principalement l'efficacité de l'administration et de la planification régionale. Nous pouvons identifier trois idées essentielles caractérisant l'école du nouveau régionalisme:
1. l'interdépendance métropolitaine;

2. la compétitivité économique métropolitaine dans le contexte de la mondialisation;

3. la viabilité du développement des régions métropolitaines.

L'argument de l'interdépendance métropolitaine ${ }^{9} \mathrm{~s}^{\prime}$ inspire beaucoup des thèses de Jane Jacobs qui insiste depuis les années 1960 sur l'importance des villes centres et de la diversité socio-économique afin d'assurer la croissance et la prospérité des régions urbaines. Cette dynamique s'obtient par la synergie entre les composantes métropolitaines, non par leur indépendance. La notion d'interdépendance métropolitaine focalise sur les relations d'interdépendance, notamment économique, entre les villes centres et les banlieues d'une même agglomération urbaine. Le nouveau régionalisme part donc du principe que la ville centrale et une grande partie de la banlieue sont en étroite interaction (Stanback, 1991). Du point de vue économique, ce paradigme regarde les économies d'agglomérations comme un lieu d'intégration des échanges économiques quotidiens et de la main-d'œuvre. Certains chercheurs tels Ledebur et Barnes (1992, 1993), Voith (1992) et Savitch et al. (1993) ont cherché à établir l'évidence statistique de l'interdépendance de villes centrales et des banlieues. Même si la plupart de ces études de corrélation ont manqué de révéler une relation de causalité concernant le rapport de dépendance entre la ville centre et la banlieue (Hill et al., 1995), ces études ont à tout le moins révélé l'intégration économique métropolitaine des banlieues et des villes centres. Pour Greenstein et Wiewel (2000), plutôt que de concevoir la ville en terme monocentrique, c'est-à-dire organisée autour d'un seul noyau central selon la vision traditionnelle de la ville, ces derniers adoptent une conception polycentrique du développement urbain qui tient compte d'une multitude de petits et moyens centres organisés autour d'un plus gros. Il faut désormais tenir compte des échanges entre toutes les composantes métropolitaines pour établir les liens d'interdépendance métropolitaine.

Le nouveau régionalisme traite abondamment de l'importance grandissante accordée au rôle des régions métropolitaines ${ }^{10}$ dans le système économique mondial (Peirce $e t$ al. 1993). Dans cette perspective, de plus en plus d'auteurs conçoivent la structure socio-économique des pays comme une constellation d'agglomérations urbaines en concurrence entre elles, produisant la majeure partie du dynamisme économique national (Barnes et Ledebur, 1997). La concurrence viendrait de l'extérieur de la région et non plus de l'intérieur. Dans la logique de la mondialisation 
et des unions économiques de libre échange, plusieurs auteurs considèrent la structure économique dans une perspective mondiale, à l'intérieur de laquelle les grandes agglomérations sont en concurrence entre elles et non plus les pays ou les nations (Omahe, 1995). Ces perspectives nous indiquent que les agglomérations sont désormais soumises à une concurrence de plus en plus intense provenant principalement de l'extérieur de la région immédiate (plutôt que de l'intérieur) et qu'elles doivent idéalement et solidairement demeurer ou devenir plus compétitives à l'échelle métropolitaine si elles ne veulent pas subir les contrecoups économiques et sociaux qu'une faible capacité concurrentielle est susceptible d'engendrer. Cette perspective insiste donc sur l'importance de renouveler l'organisation métropolitaine afin de favoriser la compétitivité métropolitaine dans le contexte de la mondialisation.

Le troisième argument essentiel du nouveau régionalisme veut que les principaux problèmes engendrés par le processus de métropolisation ne peuvent être résolus par l'action individuelle des municipalités et que des actions régionales sont nécessaires pour s'attaquer aux problèmes urbains. On aborde ainsi la question de la viabilité du développement métropolitain ${ }^{11}$. Parmi les problèmes les plus souvent cités par le mouvement du nouveau régionalisme on retrouve l'étalement urbain, les problèmes de transport, la ségrégation sociale, le déclin économique des villes centres, la fragmentation politique municipale et l'impact de la croissance urbaine sur l'environnement (Downs, 1994). Le nouveau régionalisme envisage tous ces problèmes comme étant interreliés et comme une menace à la viabilité sociale, économique, politique et environnementale. Myron Orfield (1996) est préoccupé par la stabilité sociale et fiscale des régions métropolitaines. C'est à partir de la redistribution spatiale de la pauvreté et de la richesse qu'il consacre l'essentiel de sa thèse. Pour David Rusk (1993), le véritable problème urbain des États-Unis est la ségrégation raciale et économique qui a contribué à créer une sous-classe de population dans les grands centres urbains. Pour Rusk, la fragmentation municipale maintient la ségrégation sociale. Sa thèse vise donc à démontrer que les villes centres qui sont en mesure d'étendre leurs limites territoriales par l'annexion ou la consolidation ou celles où l'on retrouve des institutions métropolitaines fortes sont aussi celles où la ségrégation sociale est plus faible et où la richesse est la mieux partagée. Rusk identifie un point de non-retour au-delà duquel les disparités entre les villes centres et les banlieues devient à toutes fins pratiques insurmontable. Pour les écologistes, l'étalement urbain est devenu un des principaux enjeux environnementaux avec l'économie, le recyclage et la réutilisation de l'eau, de l'énergie et des déchets solides. Ce mouvement se préoccupe surtout des conséquences de l'étalement urbain, de la sur-exploitation des espaces agricoles et naturels, de la pollution de l'eau et de l'air. Ils souhaitent des politiques régionales visant le contrôle de la croissance, la protection du territoire, l'établissement de ceintures vertes, la diminution du transport individuel à la faveur du transport en commun, ainsi qu'un modèle de développement beaucoup plus dense. Le tableau 3 fait la synthèse des arguments du nouveau régionalisme.

En somme, pour plusieurs contributeurs de ce courant de pensée, l'interdépendance, la compétitivité et la viabilité du développement métropolitain passent par un ensemble d'initiatives inspirées par les principes invoqués plus haut et nécessitant une approche métropolitaine. Ces approches

\section{Tableau 3. Synthèse des arguments du nouveau régionalisme}

\begin{tabular}{ll}
\hline $\begin{array}{l}\text { L'interdépendance } \\
\text { métropolitaine }\end{array}$ & - Structure polycentrique du développement (multitude de petits pôles organisés autour d'un plus gros) \\
& - Intégration économique et sociale entre les zones centrales et les banlieues d'une même région métropolitaine \\
\hline $\begin{array}{l}\text { La compétitivité } \\
\text { économique } \\
\text { métropolitaine }\end{array}$ & - Rôle des agglomérations métropolitaines dans le système économique mondial (pôles de production économique) \\
& - Concurrence économique entre les agglomérations métropolitaines \\
\hline $\begin{array}{l}\text { La viabilité } \\
\text { du développement de renouvellement de l'organisation intramétropolitaine dans le but de favoriser la compétitivité régionale } \\
\text { métropolitain }\end{array}$ & $\begin{array}{l}\text { l'étalement urbain, les problèmes de transport, la ségrégation sociale, le déclin des zones centrales, } \\
\text { régions métropolitaines }\end{array}$ \\
& - À long terme, les disparités entre les zones centrales et les banlieues compromettent le développement métropolitain \\
& Nécessité d'une approche régionale coordonnée pour faire face aux problèmes engendrés par la métropolisation \\
\hline
\end{tabular}


peuvent comprendre la création de gouvernements métropolitains ou le renforcement des instances métropolitaines actuelles, des stratégies de développement ou de promotion économique conjointes pour l'ensemble de l'agglomération, des mesures de partage de l'assiette foncière (tax base sharing), des politiques de contrôle de l'étalement urbain et de transport coordonnées, etc. Le nouveau régionalisme puise donc à une grande diversité d'approches métropolitaines et ne se limite pas à l'établissement de nouvelles structures gouvernementales ou à d'autres solutions de type structurel comme le regroupement municipal.

\section{La portée du nouveau régionalisme sur la réorganisation du pouvoir métropolitain}

Pour certains nouveaux régionalistes comme Rusk, Orfield et Peirce, une forme institutionnelle centralisée s'avère presque essentielle pour la réussite des réformes métropolitaines qu'ils envisagent à travers leurs positions respectives. L'annexion municipale ou une forme ou une autre de gouvernement métropolitain détenant une légitimité et un mandat régional serait alors de mise. Pour d'autres, comme Wallis et Dodge ${ }^{12}$, c'est dans le réseautage et la mobilisation des forces gouvernementales et non gouvernementales que réside la clé du nouveau régionalisme pour laquelle l'infrastructure civique régionale s'avère plus déterminante pour la gouvernance métropolitaine que les réformes institutionnelles.

\section{Dans les faits, le mouvement du nouveau régionalisme n'a pas jusqu'à maintenant bousculé significativement les structures de l'organisation municipale et métropolitaine aux États-Unis.}

Dans les faits, le mouvement du nouveau régionalisme n'a pas jusqu'à maintenant bousculé significativement les structures de l'organisation municipale et métropolitaine aux États-Unis. Ce que l'on remarque toutefois, c'est une plus grande préoccupation au sein des programmes et des politiques des instances gouvernementales et des organisations civiques pour les principes du nouveau régionalisme basés sur l'interdépendance métropolitaine, la compétitivité économique, et la viabilité sociale et environnementale. Le discours du nouveau régionalisme se fraye ainsi un chemin à l'intérieur des programmes habituels des institutions déjà existantes.

Faute d'imposer un nouvel ordre institutionnel métropolitain, le mouvement du nouveau régionalisme a plutôt favo- risé une certaine mobilisation d'acteurs régionaux autour des enjeux du développement et de la croissance métropolitaine dans le courant des années 1990. On observe également que cette mobilisation a davantage été initiée et soutenue par les organisations non gouvernementales que par les gouvernements locaux et centraux. Les associations de gens d'affaires, les groupes sociaux et environnementaux sont particulièrement actifs dans ce débat. Les approches préconisées par le nouveau régionalisme favorisent donc des formes de pouvoir souples, s'appuyant sur des réseaux d'acteurs gouvernementaux et non gouvernementaux plutôt que sur le regroupement municipal ou la création de nouvelles structures métropolitaines. En somme, si les principes du nouveau régionalisme tendent à se tailler graduellement une place dans l'action et les programmes des acteurs régionaux, cela s'effectue de manière très graduelle dans le respect des structures gouvernementales existantes.

Les approches préconisées par le nouveau régionalisme favorisent donc des formes de pouvoir souples, s'appuyant sur des réseaux d'acteurs gouvernementaux et non gouvernementaux plutôt que sur le regroupement municipal ou la création de nouvelles structures métropolitaines.

\section{Conclusion}

Si au Canada les plus récentes réformes métropolitaines ont surtout été l'affaire des gouvernements provinciaux, aux États-Unis, le nouveau régionalisme métropolitain est l'affaire d'une grande diversité d'acteurs. Ce mouvement repose d'ailleurs davantage sur le leadership des acteurs civiques que sur celui des acteurs gouvernementaux. Il est encore trop tôt pour faire le bilan de la portée de ce mouvement sur la réorganisation du pouvoir métropolitain, mais on peut dire cependant sans trop se tromper que ce mouvement commence à s'implanter dans les pratiques de planification, d'aménagement et de développement des institutions déjà existantes. Ainsi, si les principes d'interdépendance métropolitaine, de compétitivité économique et de viabilité du développement métropolitain continuent à se tailler une place dans les pratiques métropolitaines, il est fort à parier que cela se fera sans le recours massif aux fusions municipales tel que l'ont connu les principales régions métropolitaines de l'Ontario et du Québec ces dernières années. Dans ce sens, l'importance du rôle affirmé des gouvernements provinciaux au Canada contraste significativement avec les approches étasuniennes récentes en matière de réorganisation du pouvoir métropolitain. 


\section{Notes et références}

1 Éric Champagne est étudiant au Doctorat en études urbaines, INRS-Urbanisation, Culture et Société.

2 Plusieurs commissions d'études ont donné le ton aux grandes réformes municipales en Ontario et au Québec dont le Greater Toronto Area Task Force (1995-1996) et les efforts répétés de réorganisation métropolitaine de Montréal avec le Groupe de travail sur Montréal et sa région (1992-1993) ainsi que la Commission nationale sur les finances et la fiscalité locale (1999).

3 Ce concept existe dans la littérature en études urbaines depuis le milieu des années 1990 et a fait l'objet de numéros spéciaux dans certaines revues scientifiques dont: FRISKEN, Frances et Donald F. NORRIS (dir.) (2001). « Regionalism Reconsidered», Journal of Urban Affairs, vol. 23, n ${ }^{\circ} 5$; SAVITCH, Hank V. et Ronald K. VOGEL (2000). «New Regionalism and its Policy Agenda », State and Local Goverment Review, vol. 33, $\mathrm{n}^{\circ} 3$.

4 À ces trois institutions locales, nous pourrions ajouter les commissions scolaires. Comme nous nous intéressons surtout à la gestion des services municipaux et métropolitains généraux, nous ne traitons pas du cas des commissions scolaires dans cet article.

5 À l'exception du Connecticut et du Rhode Island, tous les États sont divisés en comtés.

6 FOSTER, Katryn A. (1997). The Political Economy of SpecialPurpose Government, Washington, D.C., Georgetown University Press.

7 Le terme conseil régional désigne indifféremment les Regional Planning Commissions et les Councils of Governments. Les conseils régionaux assument généralement le rôle de Metropolitan Planning Organization pour se conformer aux exigences des programmes fédéraux en matière d'investissements publics.

8 Deux cas d'exception sont fréquemment cités comme modèles de gouvernement ou de quasi-gouvernement métropolitain aux États-Unis. Il s'agit des cas du Metropolitan Council of the Twin Cities Area dans l'agglomération de Minneapolis au Minnesota et de celui du Metropolitan Service District (Metro) de l'agglomération de Portland en Oregon.

9 Littérature citée sur l'interdépendance métropolitaine : STANBACK, Jr., Thomas M. (1991). The New Suburbanization. Challenge to the Central City, San Francisco, Westview Press;
LEDEBUR, L.C. et W.R. BARNES (1992). «Metropolitan Disparities and Economic Growth: City Distress and the Need for a Federal Local Growth Package », Research Report on America's Cities, Washington, D.C., National League of Cities ; LEDEBUR, L.C. et W.R. BARNES (1993). «All in it Together: Cities, Suburbs and Local Economic Regions », Research Report on America's Cities, Washington, D.C., National League of Cities ; VOITH, Richard (1992). "City and Suburban Growth: Substitutes or Complements?», Business Review, (septembreoctobre), p. 21-32 ; SAVITCH, H.D., D. COLLINS, D. SETERS et J.P. MARKHAM (1993). «Ties that Bind: Central Cities, Suburbs, and the New Metropolitan Region », Economic Development Quarterly, vol. 7, no 4, p. 341-57 ; RUSK, David (1993). Cities Without Suburbs, Washington, D.C., The Woodrow Wilson Center Press ; HILL, E.W., H.L. WOLMAN et C.C. FORD III (1995). «Can Suburbs Survive Without Their Central Cities? Examining the Suburban Dependence Hypothesis », Urban Affairs Review, vol. 31, p. 147-173 ; GREENSTEIN, Rosalind et Wim WIEWEL (dir.) (2000). Urban-Suburban Interdependencies, Cambridge, MA, Lincoln Institute of Land Policy.

10 Littérature citée sur la compétitivité économique métropolitaine: PEIRCE, Neil, Curtis W. JOHNSON et John Stuart HALL (1993). Citistates. How Urban America Can Prosper in a Competitive World, Washington, D.C., Seve Locks Press ; BARNES, William R., Larry C. LEDEBUR (1997). The New Regional Economies: The U.S. Common Market and the Global Economy, Thousand Oaks, SAGE; OHMAE, Kenichi (1995). The End of the Nation State. The Rise of Regional Economies, New York, The Free Press.

11 Littérature citée sur la viabilité du développement métropolitain : DOWNS, Anthony (1994). New Visions for Metropolitan America, Washington, D.C., The Brookings Institution; ORFIELD, Myron (1996). Metropolitics: A Regional Agenda For Community and Stability, Brookings Institution Press and Lincoln Institute; RUSK, David (1993). Cities Without Suburbs, Washington, D.C., The Woodrow Wilson Center Press.

12 WALLIS, Allan D. (1993). «Governance and the Civic Infrastructure of Metropolitan Regions », National Civic Review, printemps, p. 125-139; DODGE, William R. (1992). «Strategic Intercommunity Governance Networks : Signets of Economic Competitiveness in the 1990s », National Civic Review, automnehiver, p. 403-417; DODGE, William R. (1996). Regional Excellence, Washington, D.C., National League of Cities. 